\title{
Orbital solutions for SB2 systems with a HgMn component ${ }^{\star} \star \star \star$
}

\author{
G. Catanzaro ${ }^{1, \star \star \star}$ and P. Leto ${ }^{2}$ \\ 1 INAF - Catania Astrophysical Observatory, via S. Sofia 78, 95123, Catania, Italy \\ ${ }^{2}$ Istituto di Radioastronomia del CNR, Stazione VLBI di Noto, CP 161 Noto, Italy \\ e-mail: pleto@noto.ira.cnr.it
}

Received 6 October 2003 / Accepted 27 November 2003

\begin{abstract}
From a new set of spectroscopic observations we determined orbital parameters of six SB2 systems with one or both components being HgMn stars.

We slightly refined the orbital periods for HD 32964, HD 173524, HD 174933 and HD 216494. Our results for HD 358 are in agreement with the previous literature studies. Regarding HD 33647, our orbital period is shorter than previous determinations. HD 173524 is a triple system. From the variations of the $\gamma$-velocity deduced from our and literature data, we refined the orbital period and we estimated the eccentricity of the third companion, for which we get $e \approx 0.13$ and $P_{\text {orb }}=36 \pm 3$ years. HD 191110 and HD 216494 seem to be the only synchronous SB2 within our sample. Following the hypothesis of rotational axes perpendicular to the orbital plane, we estimated the angle of the orbital plane to the line of sight. We identified in our spectra the signature of the $\mathrm{H}_{\beta}$ of the third component of HD 216494 and we highlight a slight variation of the $\gamma$-velocity due to the presence of this component.
\end{abstract}

Key words. stars: binaries: spectroscopic - stars: chemically peculiar - stars: general

\section{Introduction}

Among the Chemically Peculiar stars of the main sequence, the $\mathrm{HgMn}$ sub-group show anomalies in the intensity of mercury and manganese lines. In particular, spectral lines due to those elements are always stronger than the corresponding lines observed in normal main sequence stars.

For binarity among $\mathrm{HgMn}$ stars, there is clear evidence that the incidence of SB systems is similar to that observed in normal stars. Abt \& Snowden (1973) carried out the first systematic search for binaries among CP stars and found $43 \%$ of their sample of $\mathrm{HgMn}$ stars belong to SB system. This percentage is very similar to the incidence of binary among normal stars found by Jaschek \& Gomez (1970). Aikman (1976) increased the small sample of Abt \& Snowden (1973), raising it from 15 to 80 stars, confirming the conclusion. Floquet (1983) analyzed the catalogue of $\mathrm{HgMn}$ stars published by Schneider (1981) and concluded that $54 \%$ of these objects have variable radial velocity.

On the other hand, it has been found that double-lined binaries (SB2) are more common among HgMn than among

Send offprint requests to: $\mathrm{G}$. Catanzaro,

e-mail: gcatanzaro@ct.astro.it

* Based on observations collected at the Stellar Station "M. G. Fracastoro" of the Catania Astrophysical Observatory, Italy.

$\star \star$ Appendix A is only available in electronic form at http://www.edpsciences.org

$\star \star \star$ Guest User, Canadian Astronomy Data Centre, which is operated by the Dominion Astrophysical Observatory for the National Research Council of Canada's Herzberg Institute of Astrophysics. normal stars (Wolff \& Preston 1978; Gerbaldi et al. 1985). Wolff \& Preston (1978) suggested that this excess of SB2, and of close binaries in general, could be explained in term of a strong trend toward synchronism of rotation and revolution, and therefore to low rotational velocities. Slow rotational velocity is one of the most important constraints needed to allow the formation of chemical peculiarity in the stellar atmosphere.

The knowledge of the orbital parameters is of great importance to study the global properties of this group of stars. To deal with homogeneous data sets, we selected all the SB2 systems reported in the catalogue of $\mathrm{HgMn}$ stars compiled by Schneider (1981) (23 out of 127 stars). In this paper we present and discuss the results for the first seven orbits, including the SB2 system HD 191110 recently published in Catanzaro et al. (2003).

\section{Observations and data reduction}

Radial velocities for the program stars listed in Table A.1 have been derived from spectra obtained with two different instruments:

- the $0.9 \mathrm{~m}$ telescope of the Catania Astrophysical Observatory (OAC), which is fiber-linked to a REOSC echelle spectrograph, was used to obtain spectroscopy of the program stars in the 4810-5370 ̊ spectral region. The resolving power deduced from the lines of the Th-Ar lamp is $\approx 14000$. During 2003 the spectrograph was updated, the resolving power increased to 20000 .

- for HD 173524 and HD 216494, we also used spectra downloaded from the Canada-France-Hawaii Telescope (CFHT) archive. These spectra were obtained with the 
$3.6 \mathrm{~m}$ telescope equipped with the $f / 4$ coudé (Gecko) spectrograph. The resolving power, as measured from the Th-Ar calibration lamp, is between 60000 and 110000 .

The stellar spectra, calibrated in wavelength and with the continuum normalized to unity, were obtained using standard data reduction procedures for spectroscopic observations within the NOAO/IRAF package. The $S / N$ achieved was always above 300 for the CFHT spectra and $\approx 100$ for the OAC data.

To calculate radial velocities for each stars, we first identified all unblended spectral lines using the Kurucz (1993) lines list. For each of these lines we measured the central wavelength with a Gaussian fit of the profile and we computed the radial velocity using the classical Doppler shift formula. Finally we averaged the values obtained and computed the rms. In one spectrum of HD 173524 taken at CFHT $($ HJD = 2449968.7765) the stars were close to conjunction, so the previous procedure could not be applied. To determine the radial velocity we modeled the observed spectrum as described in Catanzaro et al. (2003). Photospheric parameters have been adopted from Adelman et al. (1998).

Systematic errors have been estimated in Catanzaro et al. (2003), $2.50 \pm 1.37 \mathrm{~km} \mathrm{~s}^{-1}$. At the beginning of 2003, because of the change in the resolving power of the spectrograph, we needed to re-evaluate the errors. We observed stars with constant and well known radial velocity: HD 107328, HD 136202, HD 161096 and HD 204867 extracted from the list of CORAVEL standard stars (Udry et al. 1999). We then computed the differences between radial velocity values from the literature and our measurements. Since those differences are smaller than $1 \mathrm{~km} \mathrm{~s}^{-1}$, we neglected them and the measured radial velocities of program stars observed in 2003 were not corrected.

The program stars with their measured radial velocities, $\sigma$ and heliocentric Julian Date are listed in Table A.1.

\section{The determination of orbital parameters}

The radial velocities for a spectroscopic binary system are:

$$
V_{\mathrm{rad}}=\gamma+K[\cos (\theta+\omega)+e \cos \omega]
$$

where $\gamma$ is the radial velocity of the center of mass, $e$ is the eccentricity of the orbit, $\omega$ is the longitude of the periastron, $\theta$ is the angular position of the star measured from the center of mass at a given instant and $K$ is the semi-amplitude of the velocity curve as given by the formula:

$K=\frac{2 \pi a \sin i}{P \sqrt{1-e^{2}}}$

where $P$ is the orbital period of the system. Orbital elements have been determined by a least-squares fitting to Eq. (1). Errors have been estimated as the variation in the parameters which increases the $\chi^{2}$ of a unit. The starting value for $P$ was evaluated using the Phase Dispersion Method (Stellingwerf 1978) as coded in the NOAO/IRAF package.

For each star, Table 3 reports the determined orbital elements, the mass and the radii ratios between components. The radii ratio for each SB2 system was evaluated using the

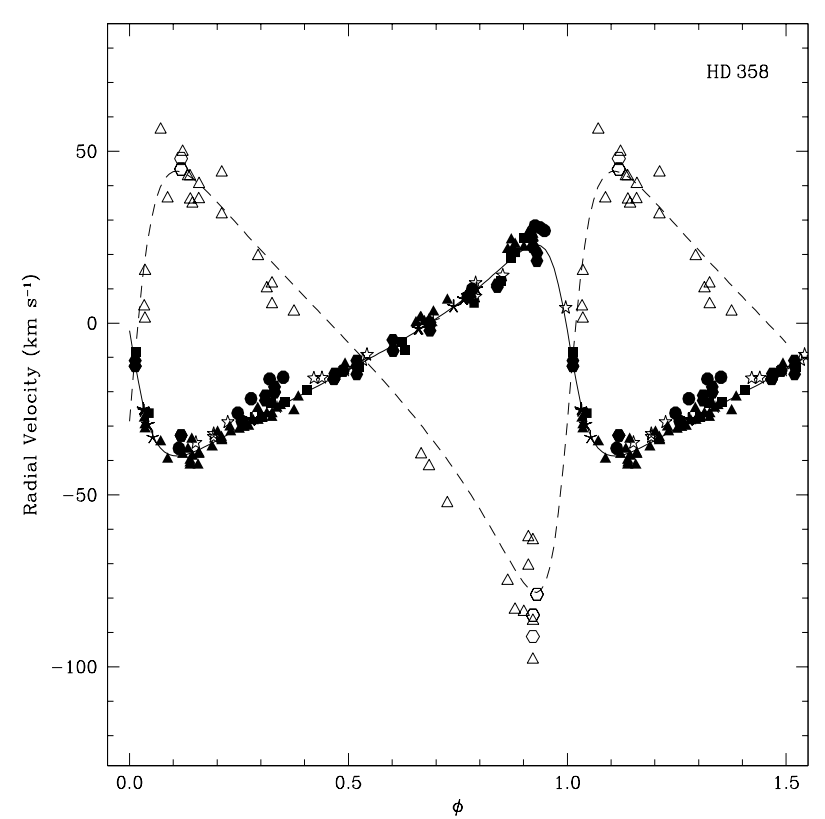

Fig. 1. Radial velocity curves of HD 358 A and B. Solid line represents the orbital solution for the primary component, while the dashed curve represents the solution of the secondary orbit. Symbols used to represent the data for HD 358 A are: stars (Abt \& Snowden 1973), filled squares (Aikman 1976), filled hexagons (Tomkin et al. 1995), filled triangles (Ryabchikova et al. 1999), asterisks (Adelman et al. 2002) and filled circles (our new measurements). To represent the data for HD 358 B we used the following open symbols: triangles (Ryabchikova et al. 1999) and hexagons (Tomkin et al. 1995).

formula given by Giménez \& Zamorano (1985)

$\log R_{A} / R_{B}=0.556 \cdot \log M_{A} / M_{B}$.

Errors on the parameters have been placed in parentheses below each quantity.

\section{Individual stars}

\section{1. $H D 358=H R 15$}

HD 358 is a bright well-known binary with a HgMn primary, whose binarity was discovered by Ludendorff (1907). As stated by Tomkin et al. (1995), the brightness of the secondary is about two magnitudes fainter than the primary. These authors and, later on, Ryabchikova et al. (1999) using high $S / N$ spectra (300 or more) and spectral resolution of $R \approx 40000$, successfully measured the secondary's radial velocities and calculated the spectroscopic orbital elements of this SB2 system. Recently Adelman et al. (2002), performed an extensive study of the spectral variability of the HgII $\lambda 3984 \AA$ line, finding the first identified spectrum variation in any $\mathrm{HgMn}$ star not due to the orbital motion of the companion. Due to the low $S / N$ of our data we did not attempt to identify the secondary's spectral lines and we proceeded in our analysis using literature data for HD $358 \mathrm{~B}$.

The least-squares fits of Abt \& Snowden (1973), Aikman (1976), Tomkin et al. (1995), Ryabchikova et al. (1999), Adelman et al. (2002) and our radial velocities using Eq. (1) give the orbital period of $96.708 \pm 0.003$ days (Fig. 1) that confirms the one found by Ryabchikova et al. (1999). 


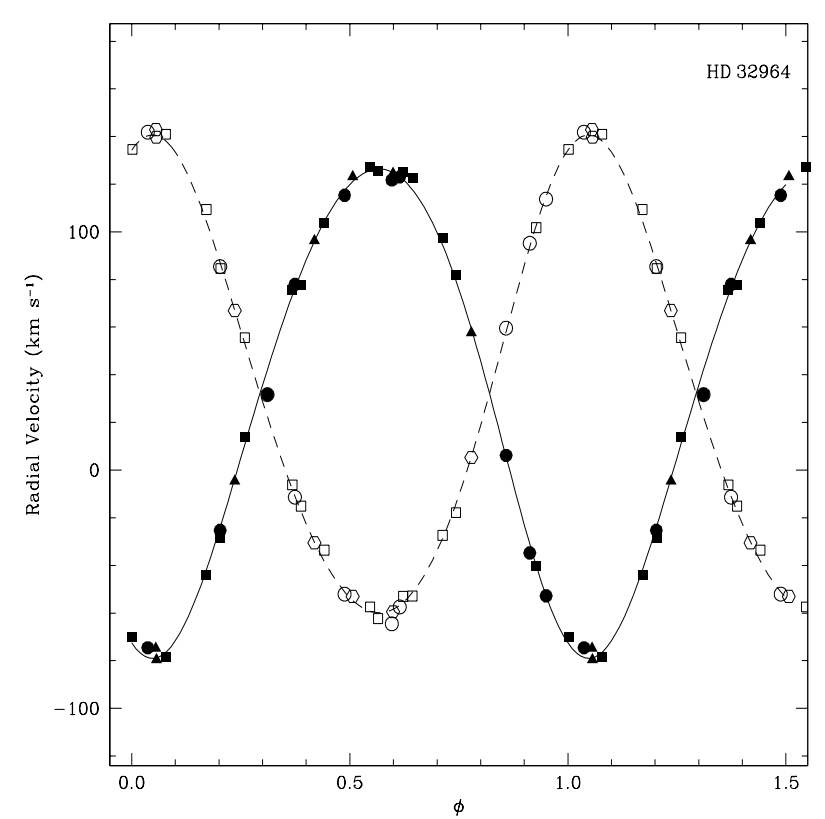

Fig. 2. Radial velocity curves of HD 32694 A and B. Solid line represents the orbital solution for the primary component, while the dashed curve represent the solution for the secondary orbit. Symbols used to represent the data are: squares for Young (1976), triangles for Yushchenko et al. $(1999,2001)$ and circles for our data. Filled symbols refer to primary component and open symbols to the secondary.

The visual orbit of $\alpha$ And has been determined by Pan et al. (1992). These authors using the Mark III Stellar Interferometre, Mount Wilson, found an inclination angle of $i=105.66 \pm$ 0.22 degrees. Combining this value with the $M \sin ^{3}(i)$ derived from our orbital solution, we got for the mass of each components $M_{A}=3.5 \pm 1.0 M_{\odot}$ and $M_{B}=1.8 \pm 0.5 M_{\odot}$. These masses are in agreement, within the errors, with those inferred by Ryabchikova et al. (1999).

\section{2. $H D 32964=H R 1657$}

HD 32964 is a well-known SB2 system since the first orbit computed by Frost \& Struve (1924) in the early 1920 s. Young (1976) used Frost \& Struve (1924) radial velocities in combination with his own data to improve the determination of the period. He found a new orbit $\left(P_{\text {orb }}=5.522731\right.$ days $)$ and pointed out that the chemical composition of the components is different. Seven new data sets were collected by Yushchenko et al. (1999, 2001), and confirmed the orbital solution computed by Young (1976).

From our spectra, taken at OAC, we collected 10 new radial velocities for both components. Our solution is in agreement with previous works. The radial velocity curves are shown in Fig. 2 and our orbital parameters are reported in Table 3.

\section{3. $H D 33647=H R 1690$}

The first radial velocity measurements of this spectroscopic binary are by Hube (1970). Wolff \& Preston (1978) measured the velocities of this SB2 system and they found a possible period

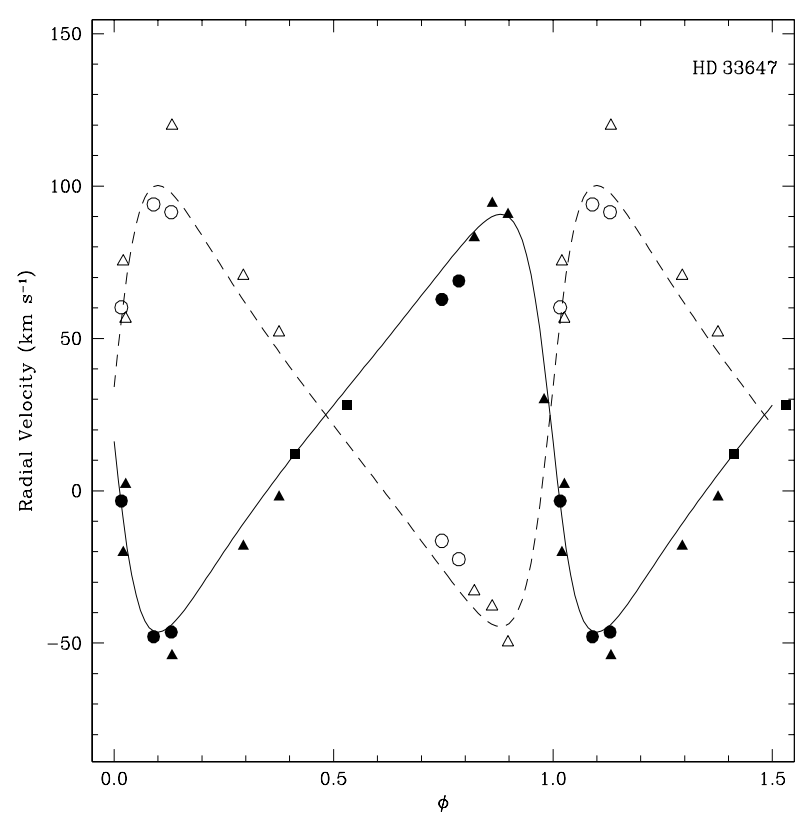

Fig. 3. Radial velocity curves of HD 33647 A and B. Solid line represents the orbital solution for the primary component, while the dashed curve represent the solution for the secondary orbit. Filled symbols are used to represent the data for HD 33647 A: squares (Hube 1970), triangles (Wolff \& Preston 1978) and circles (our data). Open symbols are used to represent the data for HD 33647 B: triangles (Wolff \& Preston 1978) and circles (our data).

of 25.01 days. A preliminary solution gave an eccentric orbit with $e=0.50$

Combining our new data with the previous, we obtained the orbital period of 24.3174 days, which is shorter than the one calculated by Wolff \& Preston (1978). Our new orbital elements are reported in Table 3 and velocity curves for this system are shown in Fig. 3.

\section{4. $H D 173524=H R 7049$}

The first orbit for this double-lined system are by Petrie (1935). His data together with those of Conti (1970) and Abt \& Snowden (1973) were used by Aikman (1976) to refine the period. He found $\approx 9.81$ days and a mass ratio of 1.18 . Afterwards, other observation were carried out by Stickland \& Weatherby (1984) and by Adelman et al. (1998).

We used all existing data plus the new velocities measured from our spectra (CFHT and OAC) to refine the period and the orbital solution. The bottom panel of Fig. 4 shows radial velocity curves for HD $173524 \mathrm{~A}$ and B; the orbital parameters are reported in Table 3.

The dispersion of velocities observed around points at the same phase has been interpreted by Adelman et al. (1998) as evidence for the presence of a third low-mass companion which revolves around the common center of mass with the spectroscopic binary. These authors gave an estimation of the possible orbital period of the third star $\left(P_{\text {orb }}=33.6 \pm 2.0\right.$ years $)$ and the semi-amplitude of the motion of the center of mass of the SB2 system $\left(K=2.6 \pm 0.4 \mathrm{~km} \mathrm{~s}^{-1}\right)$. 


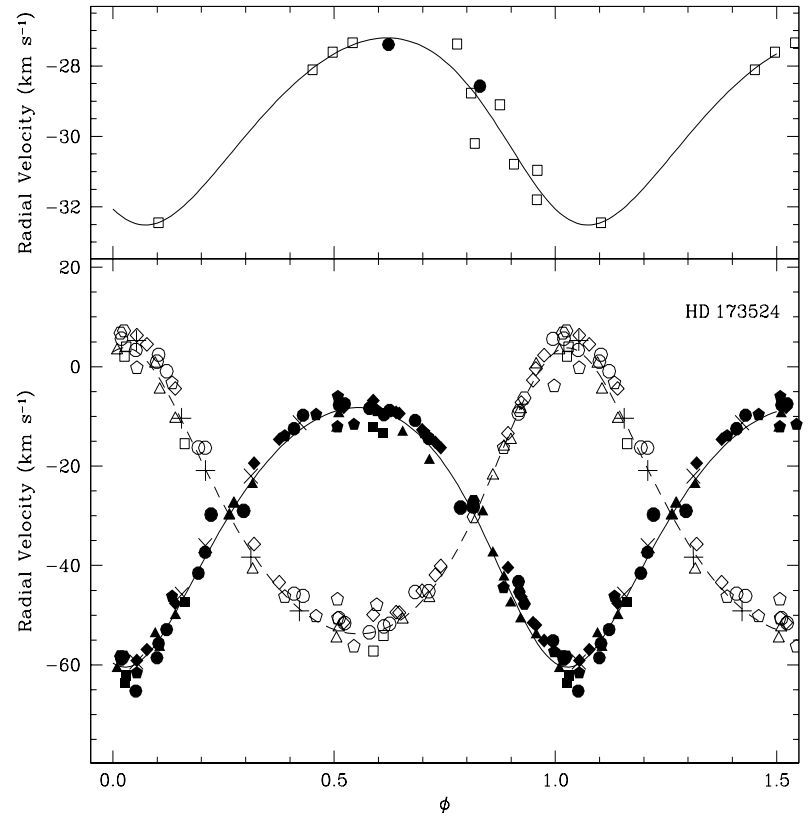

Fig. 4. Bottom panel: radial velocity curves of HD 173524 A and B. The primary component is represented by solid line and filled symbols, the secondary's by dashed line and open symbols. In particular, literature data are represented by: crosses and diagonals for respectively A and B velocities measured by Conti (1970), pentagons (Abt \& Snowden 1973), triangles (Aikman 1976), squares (Stickland \& Weatherby 1984) and rhombus (Adelman 1998). Our measurements are represented by circles (OAC spectra) and hexagons (CFHT spectra). Upper panel: velocity curve for the motion of the center of mass of HD $173524 \mathrm{~A}$ and B due to the presence of the third companion. Open squares are data taken from various literature studies and reported in Table 1, filled circles represent our observations.

Following Adelman et al. (1998), we divided all the data we have at our disposal in thirteen groups by corresponding epochs, then we computed the $\gamma$-velocity values fixing the other orbital elements equal to those we determined and reported in Table 3. Using these points (see Table 1), we found the velocity curve reported in the upper panel of Fig. 4 as a possible orbital solution for the center of mass of the SB2. These velocities have been phased with a period of $P=36 \pm 3$ years, an eccentricity $e \approx 0.13$ and $\omega \approx 146^{\circ}$. The standard deviation of the fit for the third component is $0.6 \mathrm{~km} \mathrm{~s}^{-1}$. The semi-amplitude derived from our solution is in agreement with the value given by Adelman et al. (1998).

\section{5. $H D 174933=H R 7113$}

HD 174933 is another well-studied SB2 system discovered by Meyer (1926a). Meyer found a period of 6.3624 days (Meyer 1926b), but he did not observe the secondary's lines in his spectra. A few radial-velocity measurements for the secondary have been reported by Conti (1970) and Seligman (1970). The first orbital solution for both components of this system has been computed by Ryabchikova et al. (1996), combining their own data with those published by the previous authors.

Our spectra taken at the OAC do not have a high enough signal-to-noise ratio to show the secondary's lines. Thus, as
Table 1. Heliocentric Julian Date and measured $\gamma$-velocity for HD 173524, $n$ represents the number of points used. Last two rows represent our data.

\begin{tabular}{l|ccr}
\hline \hline Epochs & $\begin{array}{c}\text { HJD } \\
(2400000.0+)\end{array}$ & $\begin{array}{c}\gamma \\
\left(\mathrm{km} \mathrm{s}^{-1}\right)\end{array}$ & $n$ \\
\hline $1928-1931$ & 26000.0 & $-28.77 \pm 0.96$ & 6 \\
1935 & 27980.0 & $-30.96 \pm 0.98$ & 14 \\
$1963-1965$ & 38800.0 & $-27.38 \pm 0.96$ & 7 \\
1966 & 39330.0 & $-30.20 \pm 0.97$ & 8 \\
1968 & 40080.0 & $-29.10 \pm 0.95$ & 5 \\
$1969-1970$ & 40500.0 & $-30.79 \pm 0.97$ & 10 \\
$1971-1974$ & 41180.0 & $-31.80 \pm 0.89$ & 3 \\
$1976-1977$ & 43100.0 & $-32.45 \pm 0.91$ & 3 \\
$1988-1989$ & 47700.0 & $-28.11 \pm 0.97$ & 10 \\
$1990-1991$ & 48300.0 & $-27.61 \pm 0.94$ & 4 \\
$1992-1993$ & 48900.0 & $-27.34 \pm 0.91$ & 3 \\
1995 & 49970.0 & $-27.39 \pm 0.95$ & 5 \\
$2001-2003$ & 52700.0 & $-28.57 \pm 0.99$ & 22 \\
\hline
\end{tabular}

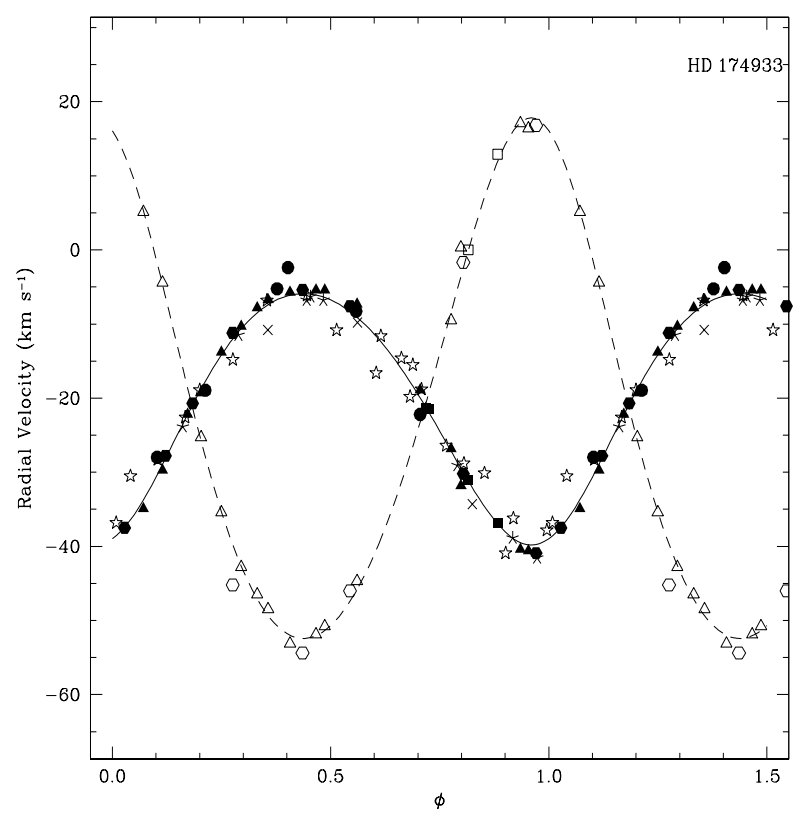

Fig. 5. Radial velocity curves of HD 174933 A and B. Solid line represents the orbital solution for the primary component, while the dashed curve represent the solution for the secondary orbit. Symbols used to represent the data for HD 174933 A are: filled squares for Seligman (1970) data, filled hexagons for Conti (1970) data, open stars for Abt \& Snowden (1973) data, asterisks for Aikman (1976) values, diagonals from Stickland \& Weatherby (1984), filled triangles from Ryabchikova et al. (1996) observations and filled circle for our measurements. Open symbols have been used to represent data for the secondary's, namely: squares for Seligman (1970), hexagons for Conti (1970) and triangles for Ryabchikova et al. (1996).

we did in the case of HD 358, we measured radial velocities only for the primary component and we used literature data for HD 174933 B. For the primary components we considered also data published by Abt \& Snowden (1973), Aikman (1976) and Stickland \& Weatherby (1984). Derived orbital parameters are reported in Table 3, while the curves are displayed in Fig. 5. 


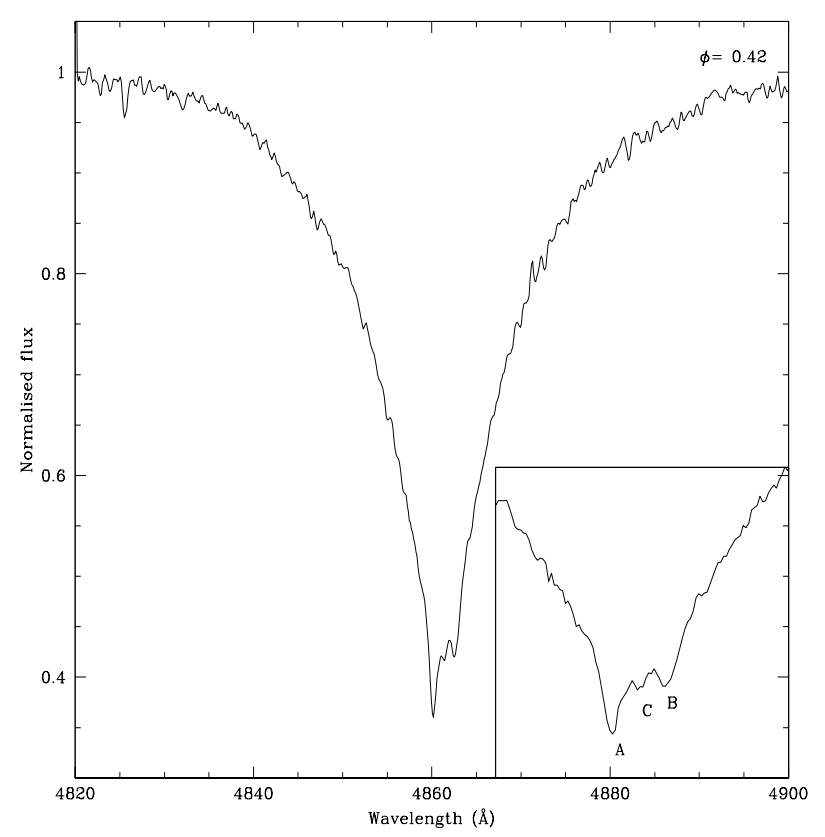

Fig. 6. Composite $\mathrm{H}_{\beta}$ of HD 216494. The contribution of the third companion is clearly visible.

\section{6. $H D 191110=H R 7694$}

HD 191110 is a SB2 system with both components being $\mathrm{CP}$ stars, precisely the primary $\mathrm{HgMn}$ and the secondary $\mathrm{Hg}$. This system has been recently studied by Catanzaro et al. (2003). The authors determined the orbital parameters (reported here again in Table 3) and they also performed a detailed abundance analysis. The rotational period is not reported in the literature but, since this system has null eccentricity, it could be considered synchronous.

In synchronous binary systems the components may be generally considered to rotate around axes perpendicular to their orbital planes and therefore the orbital inclination should correspond to the angle between the line of sight and the rotation axis of both component (Nariai 1971; Kitamura 1980).

According to these hypotheses we attempted to estimate the inclination of the orbital plane with respect to the line of sight. If the SB2 system is synchronous, the orbital period is equal to the rotational period. We then could write the following equation for the equatorial rotational velocity:

$v_{e}=\frac{2 \pi R^{*}}{P_{\text {rot }}}=\frac{2 \pi R^{*}}{P_{\text {orb }}}$.

For $\mathrm{HgMn}$ stars it is reasonable to assume $R^{*}=3 R_{\odot}$ (Gerbaldi et al. 1985; Wolff \& Preston 1978). Considering a possible indetermination in the radius of $0.5 R_{\odot}$ and $v_{e} \sin i=7 \mathrm{~km} \mathrm{~s}^{-1}$ for both components (Catanzaro et al. 2003), the inclination of the orbit ranges between 21 and 41 degrees. This result is compatible with the angle of $35^{\circ}$ reported by Guthrie (1986) in his study on the inclinations of late B-type spectroscopic binaries.

\section{7. $H D 216494=H R 8704$}

HD 216494 is one of a number of $\mathrm{HgMn}$ stars discovered in a survey of late B-type stars by Wolff \& Wolff (1974). Orbital

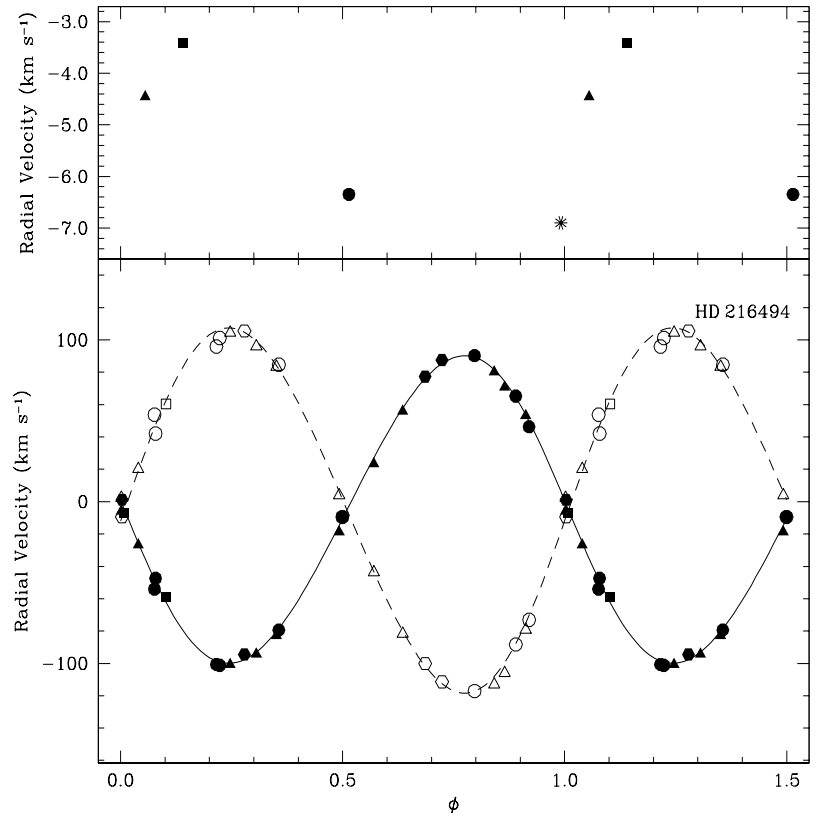

Fig. 7. Bottom panel: Radial velocity curves of HD 216494 A and B. The primary component is represented with solid line and filled symbols, the secondary's with dashed line and open symbols. In particular, triangles are data from Wolff (1974), squares are data from Aikman (1976), hexagons and circle are our measurements (CFHT and OAC, respectively). Upper panel: $\gamma$-velocity of the center of mass of the SB2 phased with the 19.25 years period (Tokovinin 1993). Asterisks are the velocity from Wolff (1974) data, triangles comes from Aikman (1976) data and squares and circles represent the $\gamma$-velocities derived from our observations (CFHT and OAC respectively).

parameters have been determined by Wolff (1974) who found a period of 3.42981 days and a mass ratio of 1.21. Other data have been collected by Aikman (1976), who did not attempt to refine the previous solution.

In an attempt to refine the orbital solution for the SB2, we observed this system for six nights at OAC and we also used four spectra downloaded from CFHT archive. Combining the measured radial velocities with those from the literature we performed a least-squares fit to Eq. (1). Results are reported in Table 3 and velocity curves are shown in Fig. 7 (bottom panel). We obtained a slightly shorter period than the result published by Wolff (1974) and a mass ratio equal, within the experimental error, to her determination.

Table 2. Heliocentric Julian Date and measured $\gamma$-velocity for HD 216494, $n$ represents the number of points used.

\begin{tabular}{l|ccc}
\hline \hline Obs. & $\begin{array}{c}\text { HJD } \\
(2400000.0+)\end{array}$ & $\begin{array}{c}\gamma \\
\left(\mathrm{km} \mathrm{s}^{-1}\right)\end{array}$ & $n$ \\
\hline Wolff (1974) & 41900.0 & $-6.90 \pm 0.98$ & 11 \\
Aikman (1976) & 42350.0 & $-4.46 \pm 0.85$ & 2 \\
CFHT & 49970.0 & $-3.41 \pm 0.55$ & 4 \\
OAC & 52600.0 & $-6.35 \pm 1.21$ & 9 \\
\hline
\end{tabular}


Table 3. Orbital parameters and their errors (quantities in parentheses). Errors are defined as the variation in the parameter which increases the $\chi^{2}$ of a unit. In the last column we report the standard deviation of the fit for both components.

\begin{tabular}{|c|c|c|c|c|c|c|c|c|c|c|c|}
\hline $\begin{array}{r}\text { Star } \\
\text { HD }\end{array}$ & $\begin{array}{c}P \\
\text { days }\end{array}$ & $\begin{array}{c}T_{0} \\
2400000+\end{array}$ & $e$ & $\begin{array}{c}\gamma \\
\mathrm{km} \mathrm{s}^{-1}\end{array}$ & $\begin{array}{c}K \\
\mathrm{~km} \mathrm{~s}^{-1}\end{array}$ & $\begin{array}{c}\omega \\
\text { degree }\end{array}$ & $\begin{array}{c}a \sin (i) \\
R_{\odot}\end{array}$ & $\begin{array}{c}M \sin ^{3}(i) \\
M_{\odot}\end{array}$ & $M_{A} / M_{B}$ & $R_{A} / R_{B}$ & $\begin{array}{l}\sigma_{(\mathrm{O}-\mathrm{C})} \\
\mathrm{km} \mathrm{s}^{-1}\end{array}$ \\
\hline $358 \mathrm{~A}$ & $\begin{array}{c}96.708 \\
(0.003)\end{array}$ & $\begin{array}{c}52597.3 \\
(0.5)\end{array}$ & $\begin{array}{l}0.54 \\
(0.04)\end{array}$ & $\begin{array}{c}-11 \\
(1)\end{array}$ & $\begin{array}{l}31 \\
(2)\end{array}$ & $\begin{array}{l}79 \\
(1)\end{array}$ & $\begin{array}{l}50 \\
(4)\end{array}$ & $\begin{array}{l}3.2 \\
(0.8)\end{array}$ & $\begin{array}{l}1.99 \\
(0.08)\end{array}$ & $\begin{array}{l}1.47 \\
(0.08)\end{array}$ & 2.4 \\
\hline $358 \mathrm{~B}$ & & & & & $\begin{array}{l}61 \\
(3)\end{array}$ & & $\begin{array}{l}99 \\
(7)\end{array}$ & $\begin{array}{l}1.6 \\
(0.3)\end{array}$ & & & 9.2 \\
\hline 32964 A & $\begin{array}{c}5.522605 \\
(0.000005)\end{array}$ & $\begin{array}{c}52567.363 \\
(0.004)\end{array}$ & $\begin{array}{l}0.088 \\
(0.004)\end{array}$ & $\begin{array}{l}32.2 \\
(0.4)\end{array}$ & $\begin{array}{c}102.7 \\
(0.8)\end{array}$ & $\begin{array}{c}159.5 \\
(0.3)\end{array}$ & $\begin{array}{l}11.16 \\
(0.09)\end{array}$ & $\begin{array}{l}2.3 \\
(0.1)\end{array}$ & $\begin{array}{l}0.98 \\
(0.02)\end{array}$ & $\begin{array}{l}0.99 \\
(0.02)\end{array}$ & 3.2 \\
\hline 32964 B & & & & & $\begin{array}{c}100.3 \\
(0.8)\end{array}$ & & $\begin{array}{l}10.90 \\
(0.09)\end{array}$ & $\begin{array}{l}2.4 \\
(0.1)\end{array}$ & & & 2.8 \\
\hline 33647 A & $\begin{array}{c}24.3174 \\
(0.0004)\end{array}$ & $\begin{array}{c}52567.0 \\
(0.1)\end{array}$ & $\begin{array}{l}0.45 \\
(0.04)\end{array}$ & $\begin{array}{l}23 \\
(2)\end{array}$ & $\begin{array}{l}69 \\
(4)\end{array}$ & $\begin{array}{l}92 \\
\text { (2) }\end{array}$ & $\begin{array}{l}30 \\
(2)\end{array}$ & $\begin{array}{c}2 \\
(1)\end{array}$ & $\begin{array}{l}1.0 \\
(0.2)\end{array}$ & $\begin{array}{l}1.0 \\
(0.3)\end{array}$ & 7.6 \\
\hline 33647 B & & & & & $\begin{array}{l}66 \\
(8)\end{array}$ & & $\begin{array}{l}28 \\
(4)\end{array}$ & $\begin{array}{c}2 \\
(1)\end{array}$ & & & 10.4 \\
\hline $173524 \mathrm{~A}$ & $\begin{array}{l}9.81053 \\
(0.00002)\end{array}$ & $\begin{array}{c}52884.31 \\
(0.02)\end{array}$ & $\begin{array}{l}0.18 \\
(0.02)\end{array}$ & $\begin{array}{c}-29.9 \\
(0.4)\end{array}$ & $\begin{array}{l}26.1 \\
(0.7)\end{array}$ & $\begin{array}{c}166 \\
(1)\end{array}$ & $\begin{array}{l}5.0 \\
(0.1)\end{array}$ & $\begin{array}{l}0.085 \\
(0.008)\end{array}$ & $\begin{array}{l}1.11 \\
(0.05)\end{array}$ & $\begin{array}{l}1.06 \\
(0.06)\end{array}$ & 2.4 \\
\hline $173524 \mathrm{~B}$ & & & & & $\begin{array}{l}28.9 \\
(0.9)\end{array}$ & & $\begin{array}{l}5.5 \\
(0.2)\end{array}$ & $\begin{array}{l}0.076 \\
(0.008)\end{array}$ & & & 2.6 \\
\hline $174933 \mathrm{~A}$ & $\begin{array}{l}6.36246 \\
(0.00002)\end{array}$ & $\begin{array}{c}52540.11 \\
(0.03)\end{array}$ & $\begin{array}{l}0.11 \\
(0.03)\end{array}$ & $\begin{array}{c}-21.0 \\
(0.4)\end{array}$ & $\begin{array}{l}17.0 \\
(0.6)\end{array}$ & $\begin{array}{l}198 \\
(2)\end{array}$ & $\begin{array}{l}2.12 \\
(0.08)\end{array}$ & $\begin{array}{l}0.06 \\
(0.01)\end{array}$ & $\begin{array}{l}2.07 \\
(0.08)\end{array}$ & $\begin{array}{l}1.50 \\
(0.07)\end{array}$ & 1.8 \\
\hline 174933 B & & & & & $\begin{array}{l}35 \\
(2)\end{array}$ & & $\begin{array}{l}4.4 \\
(0.3)\end{array}$ & $\begin{array}{l}0.03 \\
(0.01)\end{array}$ & & & 1.7 \\
\hline $191110 \mathrm{~A}^{\dagger}$ & $\begin{array}{l}9.34661 \\
(0.00002)\end{array}$ & $\begin{array}{c}52572.65 \\
(0.02)\end{array}$ & $\begin{array}{c}0 . \\
(0.02)\end{array}$ & $\begin{array}{c}-8.3 \\
(0.6)\end{array}$ & $\begin{array}{l}52 \\
(1)\end{array}$ & $\begin{array}{c}220 \\
(1)\end{array}$ & $\begin{array}{l}9.7 \\
(0.2)\end{array}$ & $\begin{array}{l}0.66 \\
(0.07)\end{array}$ & $\begin{array}{l}1.08 \\
(0.04)\end{array}$ & $\begin{array}{l}1.04 \\
(0.04)\end{array}$ & 2.1 \\
\hline $191110 \mathrm{~B}^{\dagger}$ & & & & & $\begin{array}{l}57 \\
\text { (1) }\end{array}$ & & $\begin{array}{l}10.5 \\
(0.2)\end{array}$ & $\begin{array}{l}0.61 \\
(0.05)\end{array}$ & & & 2.7 \\
\hline $216494 \mathrm{~A}$ & $\begin{array}{c}3.429616 \\
(0.000004)\end{array}$ & $\begin{array}{c}52909.150 \\
(0.007)\end{array}$ & $\begin{array}{l}0.05 \\
(0.02)\end{array}$ & $\begin{array}{l}-5 \\
(1)\end{array}$ & $\begin{array}{l}95 \\
(2)\end{array}$ & $\begin{array}{l}86.5 \\
(0.8)\end{array}$ & $\begin{array}{l}6.4 \\
(0.1)\end{array}$ & $\begin{array}{l}1.7 \\
(0.2)\end{array}$ & $\begin{array}{l}1.19 \\
(0.03)\end{array}$ & $\begin{array}{l}1.10 \\
(0.04)\end{array}$ & 2.6 \\
\hline 216494 B & & & & & $\begin{array}{c}113 \\
(2)\end{array}$ & & $\begin{array}{r}7.6 \\
(0.2) \\
\end{array}$ & $\begin{array}{r}1.4 \\
(0.1) \\
\end{array}$ & & & 5.0 \\
\hline
\end{tabular}

†rbital parameters taken from Catanzaro et al. (2003).

HD 216494 is a triple system composed of this SB2 system plus a third companion orbiting around the common center of mass in 19.25 years (Tokovinin 1993). Hubrig \& Mathys (1994) observed this system at high resolving power ( $R=100000)$. They suggested that the spectral type of the third component could be similar or slightly earlier than that of the HgMn primary. No more detail could be provided from their spectra.

We identified the $\mathrm{H}_{\beta}$ of HD $216494 \mathrm{C}$ in the spectra we obtained at the Catania Astrophysical Observatory. Figure 6 shows the composite $\mathrm{H}_{\beta}$ observed at the JD $=2452567.4115$, when the $\mathrm{A}$ and $\mathrm{B}$ components were at the orbital phase 0.42 . Measured heliocentric radial velocity for the $\mathrm{C}$ component was $3.25 \mathrm{~km} \mathrm{~s}^{-1}$. We divided all the data in four groups and, following the same procedure used for HD 173524, we computed the $\gamma$-velocities. These data are been reported in Table 2 and displayed in the upper panel of Fig. 7, where they have been phased with the orbital period of 19.25 years given by Tokovinin (1993). The small number of points did not allow us to search for an orbital solution, but a variation in the radial velocity of the center of mass of the SB2 system is clearly visible.

Manfroid \& Mathys (1985) carried out photometric observations of this star. According to their study three rotational periods are possible $(0.724 / 1.27 / 3.40$ days $)$, but given the low projected equatorial velocity observed, they concluded that the most probable period is 3.40 days. Thus, since this value is very close to the orbital period reported in Table 3, we conclude that HD 216494 could be synchronous.

Applying the same procedure as in the case of HD 191110 we attempted to estimate the orbital inclination. Abt et al. (2002) estimated a rotational velocity for the primary component $v_{e} \sin i=20 \mathrm{~km} \mathrm{~s}^{-1}$. This value is not compatible with line profiles observed in the high resolution spectra $(R=110000)$ downloaded from the CFHT archive. From these spectra we estimated an upper limit for the velocities for each components equal to $3 \mathrm{~km} \mathrm{~s}^{-1}$.

Using this value, from Eq. (4) it follows directly that $i \approx 4^{\circ}$. This inclination angle is not compatible with the high radial velocity measured for each components. Moreover, this system has been studied by Guthrie (1986), who found an inclination of the orbit equal to $54^{\circ}$, a value more compatible with the measured velocities.

Thus, the initial hypothesis of a rotational axis perpendicular to the orbital plane could be wrong. We can conclude that we are looking at the stars almost pole-on, while the true orbit is tilted of $54^{\circ}$ with respect to the line of sight. 


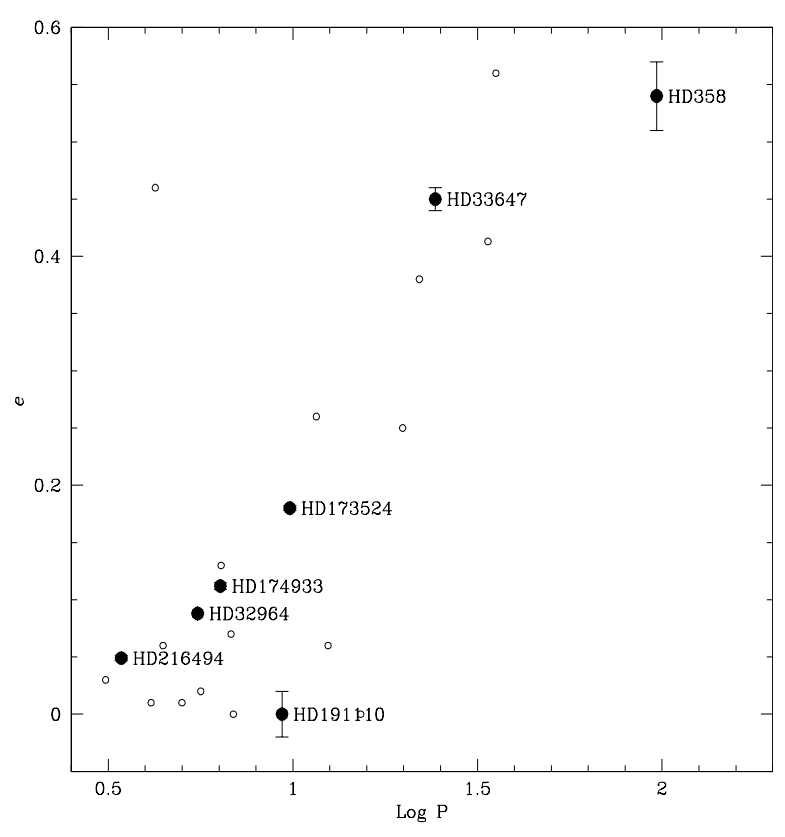

Fig. 8. The eccentricity as a function of the orbital period for our sample of SB2 system. Open circle refer to the data published by Guthrie (1986).

\section{Discussion and conclusion}

We have carried out spectroscopic observations and determined the orbital elements of six binary systems whose brightest component is a chemically peculiar star of the $\mathrm{HgMn}$ sub-group. The results are summarized in Table 3 .

In this study we first attempted to compute a possible orbital solution for the motion of the center of mass of HD $173524 \mathrm{~A}$ and B due to the presence of the third companion discovered by Adelman et al. (1998). The orbit for HD 33647 has been revised while for the other program stars orbital parameters have been slightly refined.

For HD 358, we computed the orbital solution and the masses of each component as well. The latter values have been inferred combining $M \sin ^{3}(i)$ derived by us with the inclination angle found by Pan et al. (1992). Our results agree with the ones by Ryabchikova et al. (1999).

Two SB2 systems of our program stars seem to be synchronous, HD 191110 and HD 216494. Under the hypothesis the rotational axis is perpendicular to the orbital plane and the assumption of a stellar radius equal to $3 R_{\odot}$, we attempted to estimate the inclination of their orbits. For HD 191110, we concluded the inclination angle varies in the range 21-41 degrees, in agreement with the result of Guthrie (1986). In the case of HD 216494, previous initial hypothesis fail and we concluded that the components are almost pole-on while the orbital plane is tilted by $54^{\circ}$ (Guthrie 1986) with respect to the line of sight.

HD 216494 is a triple system composed of the SB2 discussed above and a third companion revolving around the common center of gravity in 19.25 years (Tokovinin 1993). We show that the variability of the center of mass of the SB2 is compatible with the period found by Tokovinin (1993) for the third companion. Moreover, our spectra, centered on the $\mathrm{H}_{\beta}$ region, clearly show the presence of the third component.
An other important aspect to address is the membership of $\mathrm{HgMn}$ stars in multiple systems. In this study we studied two of such systems (HD 173524 and HD 216494). Another two stars are multiple systems: HD 32964 and HD 33647 (Tokovinin 1997). Thus, 4 out of 7 stars are in multiple systems, and if this percentage is representative of the sample, we could conclude that $\approx 57 \%$ of $\mathrm{HgMn}$ in SB2 systems have more than one companion.

In a review concerning the binarity among Bp-Ap stars, Gerbaldi et al. (1985) pointed out that the distribution of eccentricity for the $\mathrm{HgMn}$ sample can be considered as being the same as in any other sample of normal stars. The only difference seems to be a slight bimodal distribution for $0.4<e<0.5$, which is less in the normal star sample. Our results reported in Fig. 8, together with those by Guthrie (1986), confirm this behavior. This diagram shows that circular orbits $(e=0)$ do exist for a wide distribution of orbital periods, up to $P \approx 10$ days.

Due to the small number of systems observed to date, we do not apply other statistical considerations.

Acknowledgements. This research has made use of the SIMBAD database, operated at CDS, Strasburg, France. We wish to thank the referee, Dr. T. Ryabchikova, for her useful comments that helped us to improve the quality of our manuscript.

\section{References}

Abt, H. A., Levato, H., \& Grosso, M. 2002, ApJ, 573, 359

Abt, H. A., \& Snowden, M. S. 1973, ApJS, 25, 137

Adelman, S. J., Gulliver, A. F., Kochukhov, O. P., \& Ryabchikova, T. A. 2002, ApJ, 575, 449

Adelman, S. J., Ryabchikova, T. A., \& Davydova, E. S. 1998, MNRAS, 297, 1

Aikman, G. C. L. 1976, Publ. Dom. Astrophys. Obs., 14, 379

Catanzaro, G., Leone, F., \& Leto, P. 2003, A\&A, 407, 669

Conti, P. 1970, ApJ, 160, 1077

Floquet, M. 1983, in Les journées de Strasbourg, Vème Réunion, Observatoire de Strasbourg, 83

Frost, E. B., \& Struve, O. 1924, ApJ, 60, 313

Gerbaldi, M., Floquet, M., \& Hauck, B. 1985, A\&A, 146, 341

Giménez, A., \& Zamorano, J. 1985, Ap\&SS, 114, 259

Guthrie, B. N. G. 1986, MNRAS, 220, 559

Hube, D. P. 1970, Mem. R. Astr. Soc., 72, 233

Hubrig, S., \& Mathys, G. 1994, Poster paper presented at the JD No. 12 of the 22nd IAU General Assembly

Jaschek, C., \& Gomez, A. E. 1970, PASP, 82, 809

Kitamura, M. 1980, Ap\&SS, 68, 283

Kurucz, R. L. 1993, A new opacity-sampling model atmosphere program for arbitrary abundances. In Peculiar versus normal phenomena in A-type and related stars, IAU Colloq. 138, ed. M. M. Dworetsky, F. Castelli, \& R. Faraggiana, ASP Conf. Ser., 44, 87

Ludendorff, H. 1907, Astron. Nachr., 176, 327

Manfroid, J., \& Mathys, G. 1985, A\&AS, 59, 429

Meyer, W. F. 1926a, Lick Obs. Bull., 381, 165

Meyer, W. F. 1926b, PASP, 38, 396

Nariai, K. 1971, PASJ, 23, 529

Pan, X., Shao, M., Colavita, M. M., et al. 1992, ApJ, 384, 624

Petrie, R. M. 1935, Publ. Dom. Astrophys. Obs., 6, 285 
Ryabchikova, T. A., Malanushenko, V. P., \& Adelman, S. J. 1999, A\&A, 351, 963

Ryabchikova, T. A., Zakharova, L. A., \& Adelman, S. J. 1996, MNRAS, 283, 1115

Tomkin, J., Pan, X., \& McCarthy, J. K. 1995, AJ, 109, 780

Tokovinin, A. A. 1997, A\&AS, 124, 75

Tokovinin, A. A. 1993, Sov. Astron. Lett., 19, 383

Schneider, H. 1981, A\&AS, 44, 137

Seligman, C. E. 1970, PASP, 82, 128

Stellingwerf, R. F. 1978, ApJ, 224, 953

Stickland, D. J., \& Weatherby, J. 1984, A\&AS, 57, 55
Udry, S., Mayor, M., \& Queloz, D. 1999, Precise Stellar Radial Velocities, IAU Colloq. 170, ed. J. B. Hearnshaw, \& C. D. Scarfe, ASP Conf. Ser., 185, 367

Wolff, S. C., \& Preston, G. W. 1978, ApJS, 37, 371

Wolff, S. C., \& Wolff, R. J. 1974, ApJ, 194, 65

Wolff, R. J. 1974, PASP, 86, 173

Young, A. 1976, PASP, 88, 275

Yushchenko, A. V., Gopka, V. F., Khokhlova, V. L., \& Tomkin, J. 2001, IBVS, 5213

Yushchenko, A. V., Gopka, V. F., Khokhlova, V. L., Musaev, F. A., \& Bikmaev, I. F. 1999, Astron. Lett., 25, 453 
G. Catanzaro and P. Leto: Orbital solutions for SB2 systems with a HgMn component, Online Material p 1

\section{Online Material}


G. Catanzaro and P. Leto: Orbital solutions for SB2 systems with a HgMn component, Online Material p 2

\section{Appendix A: Measured radial velocities}

Table A.1. Heliocentric Julian Date and measured radial velocities for program stars. $n$ represents the number of spectral lines used for the velocities determination.

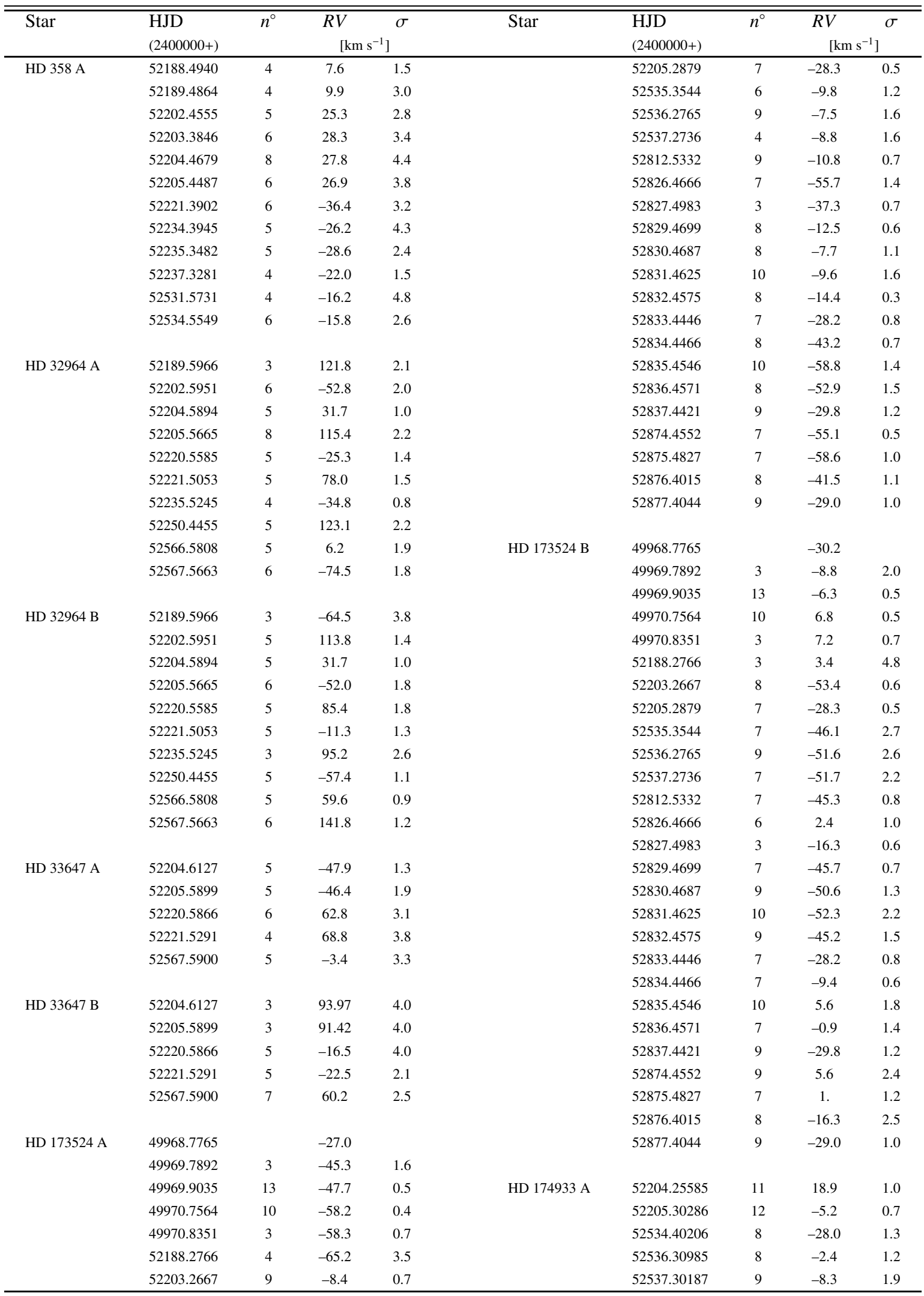


G. Catanzaro and P. Leto: Orbital solutions for SB2 systems with a HgMn component, Online Material p 3

Table A.1. continued.

\begin{tabular}{|c|c|c|c|c|c|c|c|c|c|}
\hline \multirow[t]{2}{*}{ Star } & \multirow{2}{*}{$\begin{array}{l}\text { HJD } \\
(2400000+)\end{array}$} & $n^{\circ}$ & $R V$ & $\sigma$ & \multirow[t]{2}{*}{ Star } & \multirow{2}{*}{$\begin{array}{l}\text { HJD } \\
(2400000+)\end{array}$} & \multirow[t]{2}{*}{$\overline{n^{\circ}}$} & $R V$ & \multirow[t]{2}{*}{$\sigma$} \\
\hline & & \multicolumn{3}{|c|}{$\left[\mathrm{km} \mathrm{s}^{-1}\right]$} & & & & {$\left[\mathrm{km} \mathrm{s}^{-1}\right]$} & \\
\hline \multirow[t]{9}{*}{ HD $216494 \mathrm{~A}$} & 49968.8933 & 15 & 77.3 & 0.6 & HD $216494 \mathrm{~B}$ & 49968.8933 & 10 & -100.1 & 0.8 \\
\hline & 49969.9816 & 10 & 1.2 & 0.5 & & 49969.9816 & 10 & -9.5 & 0.6 \\
\hline & 49970.9265 & 15 & -94.5 & 0.5 & & 49970.9265 & 9 & 105.6 & 0.5 \\
\hline & 52202.3760 & 8 & 46.3 & 2.1 & & 52202.3760 & 3 & -73.0 & 1.6 \\
\hline & 52237.2080 & 9 & -54.1 & 1.9 & & 52237.2080 & 9 & 53.8 & 2.7 \\
\hline & 52531.5161 & 9 & 65.2 & 1.9 & & 52531.5161 & 7 & -88.3 & 3.8 \\
\hline & 52566.4595 & 5 & -47.5 & 1.0 & & 52566.4595 & 7 & 42.1 & 4.2 \\
\hline & 52567.4115 & 7 & -79.4 & 1.7 & & 52567.4115 & 4 & 84.7 & 3.0 \\
\hline & 52906.4612 & 7 & -100.7 & 0.5 & & 52906.4612 & 6 & 95.9 & 2.2 \\
\hline
\end{tabular}

